\title{
CONTENT-BASED IMAGE RETRIEVAL USING SALIENT BOUNDARY AND CENTROID-RADII MODEL
}

Qing Wang ${ }^{1}$, Haijian $\mathrm{Ye}^{1, *}$,Yan Wang ${ }^{2}$, Hua Zhang ${ }^{1}$

${ }^{1}$ College of Information and Electrical Engineering, China Agricultural University, No. 17, Tsinghua East Road, Haidian, Beijing, 100083, China

2 Ocean College, Hebei Agricultural University, No. 52, East Part of Hebei Street, Qinhuangdao City, Hebei Province, 066003, China

"Corresponding author, Address: P. O. Box 147, College of Information and Electrical Engineering, China Agricultural University, No. 17 Tsinghua East Road, Haidian, Beijing, 100083, China, Tel: +86-10-62737188, Fax:+86-10-62736401,Email: hjye@cau.edu.cn

Abstract: In view of the instability and low efficiency of the present image retrieval method, especially for simple image comparison with some salient shapes, a new image retrieval algorithm based on salient closed boundary is presented. Firstly, the Canny operator is performed to detect edges. Secondly, the ratio contour is used to extract the most salient closed boundary of some shape from the image. Finally, the similarities are measured by feature vector of the salient closed boundary based on the centroid-radii model. Preliminary experimental results demonstrate that the proposed method is quite suitable for many professional image retrieval systems and has a good performance in both retrieval efficiency and effectiveness.

Key words: content based image retrieval, salient closed boundary, radio contour, centroidradii model

\section{INTRODUCTION}

Due to the fast development of digital imaging and networking technologies, Content-Based Image Retrieval (CBIR) has emerged as an important area of research in computer visual and multimedia computing. CBIR retrieves images from the database similar to the query image by the

Please use the following format when citing this chapter:

Wang, Q., Ye, H., Wang, Y. and Zhang, H., 2009, in IFIP International Federation for Information Processing, Volume 294, Computer and Computing Technologies in Agriculture II, Volume 2, eds. D. Li, Z. Chunjiang, (Boston: Springer), pp. 853-860. 
image characteristics such as color, texture, shape or any combination of these features. According to the human vision system theory, people tend to evaluate or understand image by its shape, namely shape-based image retrieval, which is an intuitive and useful way to make use of image's information. To find out the image shape's features close enough to the actual perceptive trait of human eyes is not a simple task. Therefore shapebased image retrieval is one of the most challenging subjects within contentbased image retrieval.

As key technology of image retrieval system, image retrieval method is the main content of study on the systematic development of image retrieval system. Indeed it doesn't merely affect the results of image retrieval directly but also determines the working efficiency and some other performances of the whole system to a great extent. Therefore, it has important significance in image retrieval algorithm's study. With the quick growth in the number of image information, it is urgent to effectively retrieve a great deal image information.

Yet, we find there are massive images with some salient shapes in, especially in a variety of professional fields such like the monitoring of diseases, insect pests and crop condition monitoring in the agricultural field, as well as the footprint comparing in the process of investigating and solve a case and so on. This kind of images is quite common. The common characteristics of those images are they have outstanding simplicity compared to general scene image, their content are usually comparatively simple and have shapes with salient boundary.

According to the characteristics of this kind of images, this paper proposes a new retrieval algorithm for images based on the shape's salient closed boundary. The basic idea of this algorithm is to extract the most salient closed boundary of some shape from the image by the ratio contour, and then the similarities are measured by the selected boundary's shape descriptor (feature vector) which is based on centroid-radii model. Experimental results demonstrate that the proposed method has a good performance in both retrieval efficiency and effectiveness.

\section{EDGE EXTRACTION}

\subsection{Edge detection}

Edge detection, as the primary condition of the image features extraction, is the first step of all kinds of image segmentation based on edge. The features extraction of the shape can be accurate and meaningful only with a flawless edge detection of the image. 
In this paper, we use the Canny operator, which is a relatively new edge detection operator with good detection performance and has been widely applied in many scopes, to extract edges from image called "fragments". The Canny algorithm is an optimal edge detection method based on a specific mathematical model for edges. According to the edge detecting theory and algorithm, Canny operator has been compared with Sobel operator, Prewitt operator, Robert operator and LoG operator through edge-detection experiments by programming (Bai $X, 2007)$. The experimental results indicate that the edge-detection performance of Canny operator within a professional image retrieval system is better than other edge operators, especially under the situation of noises of images the Canny operator has both differencing and smoothing effects and the later is useful to reduce noise in resulting fragments.

\subsection{Salient closed boundary extraction (Wang S et al., 2005)}

All the so-called edges gotten from edge detection by Canny algorithm in fact are only a set of boundary fragments, not the meaningful boundaries what we really want. In order to represent an image, a boundary of the shape with the largest saliency is needed. Shape is usually a polygonal or irregular region with closed boundary composed of multiple straight or curve lines. Ratio contour (RC) is adopted to extract salient closed boundaries from noisy images.

The ratio-contour algorithm encodes the Gestalt laws of closure, proximity and continuity in the boundary-saliency measure based on the relative gap length and average curvature when connecting fragments to form a closed boundary. Closure requires that the boundary be a cycle. Proximity requires the gap between two neighboring fragments to be small. Continuity requires the resulting boundary to be smooth. In RC, all the arcs are constructed to be smooth curve segments to achieve good boundary continuity. Let $q(t)=(x(t), y(t)), \quad t \in[0, L(B)]$ be the arc-length parameterized representation of a valid closed boundary $B$ formed by alternately connecting a sequence of arcs and fragments, where $L(B)$ is the boundary length. We know that $\mathrm{q}(\mathrm{L}(\mathrm{B}))=\mathrm{q}(0)$ as the boundary is closed.

In $\mathrm{RC}$, the boundary-cost $R(B)$ (negatively related to the boundary saliency/desirability, e.g., $\left.\phi(B)=e^{-R(B)}\right)$ is defined as:

$$
R(B)=\frac{\int_{0}^{L(B)}\left[\sigma(t)+\lambda k^{2}(t)\right] d t}{L(B)}
$$


where $\sigma(t)=1$ if $q(t)$ is on a gap-filling arc and $\sigma(t)=0$, otherwise. $k(t)$ is the curvature of the boundary at $q(t)$.

Since all the fragments are of zero length, they have no direct contribution to this cost. In the numerator of (1), the first term $\int_{0}^{L(B)} \sigma(t) d t$ makes it biased to a boundary with longer detected arcs and shorter gap-filling arcs. This reflects the preference of better proximity.

The second term $\int_{0}^{L(B)} k^{2}(t) d t$ reflects the favor of smoother boundaries, or better continuity. The denominator normalizes the cost by the boundary length $L(B)$ to avoid a bias to shorter boundaries. $\lambda>0$ is a regularization factor that balances the proximity and continuity in the cost function. From (1), boundary-cost $R(B)$ associate the length and the curvature of all gaps of boundary B. So the most salient closed boundary is the boundary with minimum boundary-cost, that is an alternate cycle with minimum cycle ratio namely Minimum Ratio Alternate (MRA) cycle.

The ratio contour algorithm for finding an MRA cycle in a solid-dashed (SD) graph $\mathrm{G}$ consists of three steps:

1). Setting the weight and length of solid edges in G to zero. Since the weight and length of any edge in the constructed SD graph G are usually nonzero, so in this step we transform the edge weights and lengths in G so that all solid edges have zero weight and length without changing the MRA cycle.

2). Detecting negative-weight alternate (NWA) cycles. By this step we reduce the problem of finding an MRA cycle in an SD graph $G$ to the problem of finding an alternate cycle with negative total edge weight in the same graph $G$ by searching for an appropriate transformation of the edge weights in G that preserves the MRA cycle but where the MRA cycle has a cycle ratio of zero.

3). Finding minimum weight perfect matchings (MWPM) to get the MRA cycle. The problem of detecting an NWA cycle in an SD graph where all the solid edges have zero weight and length can be reduced to the problem of finding an MWPM in the same graph. A perfect matching in $G$ denotes a subgraph of $G$ that contains all the vertices in $G$, but where each vertex only has one incident edge.

Fig. 1 illustrates the operation of the most-salient closed boundary extraction implementation on a sample image of black cutworm from the agricultural pests image database. 
Radii Model

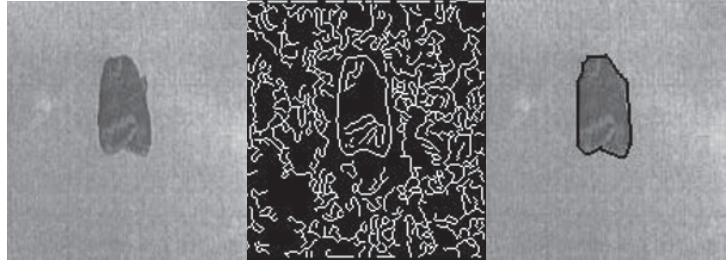

(a) (c)

Fig. 1. (a) A sample image; (b) Output of edge detection; (c) The extracted salient boundary using RC.

\section{FEATURE DESCRIPTION BASED ON THE SALIENT CLOSED BOUNDARY}

In this paper, we adopt the centroid-radii model (CRM) (K. L. Tan et al., 2000) to represent the most-salient closed boundary feature. In the centroidradii model, lengths of a shape's radii from its centroid at regular intervals are captured as the shape's descriptor (see Fig. 2). More formally, let $\theta$ be the regular interval (measured in degrees) between radii. Then, the number of intervals is given by $k=[360 / \theta]$. Furthermore, we suppose that the intervals are taken anticlockwise starting from the $\mathrm{x}$-axis direction. Thus, the shape descriptor can be represented as a vector $\left(l_{0}, l_{\theta}, l_{2 \theta}, \ldots, l_{(k-1) \theta}\right)$ where $l_{i \theta}(0 \leqslant i \leqslant(k-1))$ is the $(i+1)$ th radius from the centroid to the boundary of the shape. With sufficient number of radii, dissimilar shapes can be differentiated from each other.
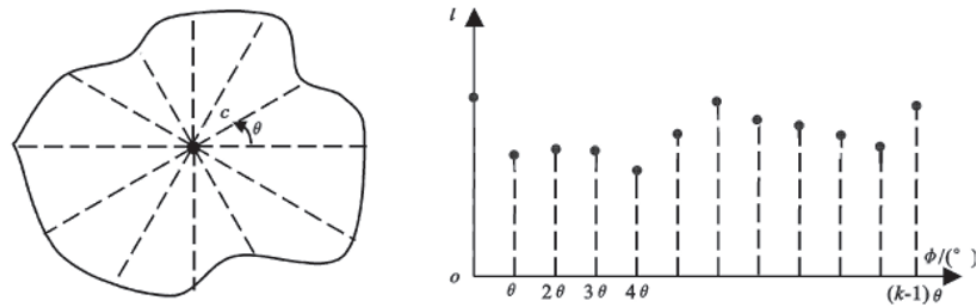

Fig. 2. The centroid-radii modeling of shape.

\section{SIMILARITY MEASURE}

Similarity measure is a key step to achieve good results of an image retrieval system. There are various formulas for distance calculation between the feature vectors and the result of the similarity measure is closely linked 
to which kind formula is adopted. A fixed image similarity measure cannot meet the need to adapt to different focuses of attention of different users.

It could be argued that we should choose different calculation methods for different feature vectors. There are many methods of image similarity measurement such as all kinds of distance measures, statistics methods and non-geometry similarity measure ways. Nowadays, commonly used formulas for distance calculation in content based image retrieval include: Minkowsky Distance, Manhattan Distance, Euclid Distance, Weighted Euclid Distance, Mahalanobis Distance, Quadratic Distance and they all have their respective appropriate application situations.

Considering the characteristics of the image we deal with and the retrieval efficiency of the image retrieval system, this paper adopts Manhattan Distance (2) to calculate the similarity between the query image and the images in the database.

$$
D(X, Y)=\sum_{i=1}^{n}\left|x_{i}-y_{i}\right|
$$

In (2), $x_{i}$ and $y_{i}$ are corresponding elements of feature vectors participated in matching, $n$ is the number of elements of the feature vector. $D(X, Y)$ is the similarity distance. Here the smaller the similarity distance, the better the similarity. In order to embody the different importance of the different feature for psychological recognizing and matching, the Manhattan Distance could be adjusted by weighting parameter.

Suppose $\mathrm{P}$ is the query image, $\mathrm{Q}$ is an arbitrary image in image database, the centroid-radii vector of the salient shape boundary of $\mathrm{P}$ and $\mathrm{Q}$ are respectively:

$$
\begin{aligned}
& L_{p}=\left(l_{p 0}, l_{p \theta}, l_{p 2 \theta}, \ldots, l_{p(k-1) \theta}\right) \\
& L_{q}=\left(l_{q 0}, l_{q \theta}, l_{q 2 \theta}, \ldots, l_{q(k-1) \theta}\right)
\end{aligned}
$$

then the Manhattan Distance between $\mathrm{P}$ and $\mathrm{Q}$ is:

$$
D(P, Q)=\sum_{i=0}^{k-1}\left|l_{p i \theta}-l_{q i \theta}\right|
$$

The similarity is measured by the distance value $D$ using (3), where $l_{p i \theta}$ and $l_{q i \theta}$ is the centroid-radii of both $\mathrm{P}$ and $\mathrm{Q} . k$ as the number of the centroid-radii is determined by $\theta$ the regular interval between radii, and $\theta$ can be determined by the experiments or relevance feedback.

\section{PRELIMINARY EXPERIMENTAL RESULTS}

In this section, we demonstrate the performance of our method using an 
experiment. The aim of this experiment is to evaluate our algorithm and compare it with the feature vector based retrieval image algorithm. For our results to be relevant, we used the same hardware environment running on the Windows XP operating system. Preliminary experiments were done on agricultural pests image database. Precision and recall are used to evaluate the efficiency and effectiveness of the algorithm. Precision is the ratio of the number of images related to the query image retrieved by the system to the total number of images retrieved automatically by the system. Recall is the ratio of the number of images related to the query image retrieved by the system to the number of all images related to the query image in the database.

Fig. 3 shows the 10 sample images used as query images to be retrieved in the database. Fig. 4 shows a graph illustrating retrieval precision and recall of the two methods used in image retrieval system on agricultural pests image database.

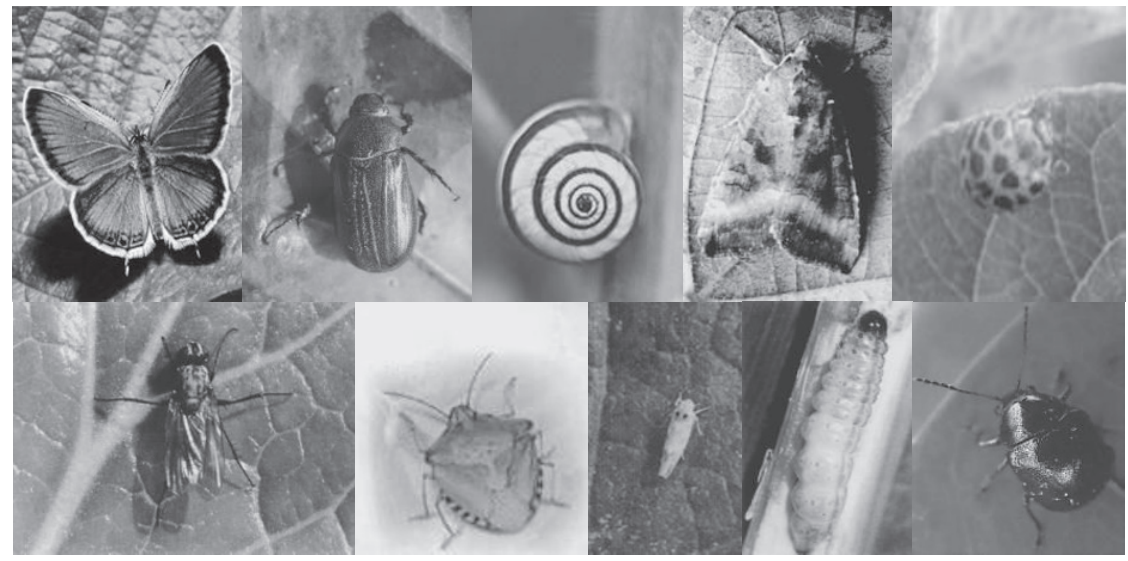

Fig. 3. The sample images used to be retrieved in the database.

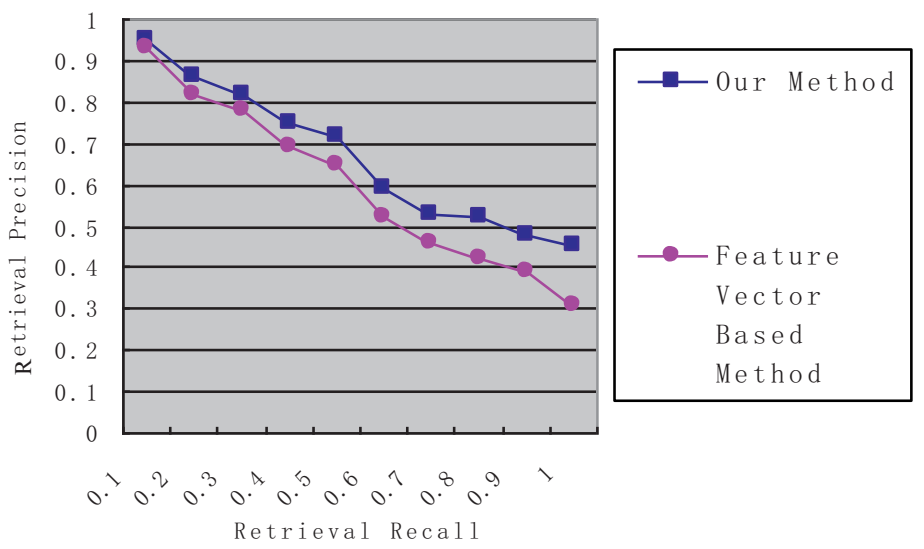

Fig. 4. Retrieval precision and recall of the two methods. 
As be seen from Fig. 4, the preliminary experimental results show that the proposed retrieval method has better retrieval precision and recall. Moreover, there are two factors to be worth notice for precision and recall statistics. One is the structure of the image database and the other is the subjective judgment of human's eyes for image similarity.

\section{CONCLUSION}

With our new image retrieval algorithm, based on shape's salient closed boundary, we are aiming at a flawless image retrieval of relatively simple images including some shapes with salient boundary. Compared to the conventional image retrieval methods, a simple shape feature representation focused on salient closed boundary has been used in this algorithm. Similarities were measured by feature vector of the salient closed boundary based on the centroid-radii model. Experimental results show that our method used in many professional image retrieval systems has a better performance in terms of efficiency and effectiveness.

\section{REFERENCES}

Bai Xue. Study on the Algorithm of Footprint Comparing [D]. Beijing: China Agricultural University, 2007, 18 23(in Chinese)

Gondra I, Heisterkamp D R. Content-based Image Retrieval with the Normalized Information Distance. Computer Vision and Image Understanding, 2008

Han Jun Wei, Guo Lei. A Shape-Based Image Retrieval Method Using Salient Edges. Image Communication, 2003, 18: 141 156

Kong Xiaodong, Luo Qingshan, Zeng Guihua, Lee M H. A New Shape Descriptor Based on Centroid-Radii Model and Wavelet Transform. Optics Communications, 2007, 273: 362 366

Tan K L, Ooi B C, Thiang L F. Indexing Shapes in Image Databases Using the Centroid Radii Model. Data and Knowledge Engineering, 2000, 32(3): 271 289

Wang S, Kubota T, Siskind J M, et al. Salient Closed Boundary Extraction with Ratio Contour. IEEE Transactions on Pattern Analysis Machine Intelligence, 2005, 27(4): 546 561

Wang S, Stahl J, Bailey A, Dropps M. Global Detection of Salient Convex Boundaries. International Journal of Computer Vision, 2007, 71(3): 337 359

Zeng Zhiyong, Zhang Xuejun, Zhou Lihua. A Novel Image Retrieval Algorithm Based on Salient Closed Boundary. Computer Science, 2006, 33(8): 221 224(in Chinese) 